\title{
Mass effects in the Higgs-gluon coupling: boosted vs. off-shell production
}

\author{
Malte Buschmann, ${ }^{a, b}$ Dorival Gonçalves, ${ }^{b}$ Silvan Kuttimalai, ${ }^{b}$ Marek Schönherr, ${ }^{b}$ \\ Frank Krauss ${ }^{b}$ and Tilman Plehn ${ }^{a}$ \\ ${ }^{a}$ Institut für Theoretische Physik, Universität Heidelberg, \\ Philosophenweg 16, 69120 Heidelberg, Germany \\ ${ }^{b}$ Institute for Particle Physics Phenomenology, \\ Department of Physics, Durham University, \\ DH1 3LE, Durham, U.K. \\ E-mail: m.s.a.buschmann@durham.ac.uk, dorival.goncalves@durham.ac.uk, \\ s.s.kuttimalai@durham.ac.uk, marek.schoenherr@durham.ac.uk, \\ frank.krauss@durham.ac.uk, plehn@uni-heidelberg.de
}

ABSTRACT: In the upcoming LHC run we will be able to probe the structure of the loopinduced Higgs-gluon coupling through kinematics. First, we establish state-of-the-art simulations with up to two jets to next-to-leading order including top mass effects. They allow us to search for deviations from the low-energy limits in boosted Higgs production. In addition, the size of the top mass effects suggests that they should generally be included in Higgs studies at the LHC. Next, we show how off-shell Higgs production with a decay to four leptons is sensitive to the same top mass effects. We compare the potential of both methods based on the same top-Higgs Lagrangian. Finally, we comment on related model assumptions required for a Higgs width measurement.

KeYwords: QCD Phenomenology, Phenomenological Models

ARXIV EPRINT: 1410.5806 


\section{Contents}

1 Higgs-top sector at the LHC 1

2 Top mass effects in Higgs rates 3

$\begin{array}{lll}3 & \text { Boosted Higgs production } & 7\end{array}$

4 Off-shell Higgs production $\quad 10$

$\begin{array}{lll}5 & \text { Width measurement } & 14\end{array}$

$\begin{array}{llr}6 & \text { Summary } & 15\end{array}$

\section{Higgs-top sector at the LHC}

The recent discovery of a light, narrow, and likely fundamental Higgs boson [1-7] makes studies of the properties of this new particle one of the key tasks of the upcoming LHC run. While the Higgs coupling structure in the Higgs-gauge sector can be extracted from precise tree-level information, our understanding of Higgs couplings to fermions largely relies on loop-induced couplings. This is obvious when we look at our currently very limited and model-dependent understanding of the top Yukawa coupling [8-24]. Its measurement from associated Higgs and top pair production with a proper reconstruction of the three heavy states is challenging [25-37].

In generic models for physics beyond the Standard Model [38] the effective gluongluon-Higgs vertex will receive contributions from dimension-6 operators proportional to $H^{2} G^{\mu \nu} G_{\mu \nu}$ and $H^{2} \bar{Q}_{L} \tilde{H} t_{R}$. One example of new physics which can generate sizable perturbative corrections to the Higgs-gluon coupling are light supersymmetric top squarks [62-68]. Because of the non-decoupling structure of the Standard Model with Yukawa couplings we can integrate out the top quark in the low-energy limit, which describes the interactions between gluons and any Higgs bosons in a simple effective Lagrangian [39-42]. For the top quark this effective Lagrangian provides a very good prediction of the inclusive Higgs production rate with at most $\mathcal{O}(10 \%)$ deviations in typical inclusive distributions for $g g \rightarrow H$ production [43-48]. On the other hand, the same description fails spectacularly once the process becomes sensitive to specific kinematic features, for instance for Higgs pair production [49-51]. Similarly, it fails for kinematic observables which generate large momentum scales, such as off-shell Higgs production with large invariant masses or the production of Higgs bosons with large transverse momenta. In these cases, the top quark cannot be integrated out anymore, since the cross section does not only include ratios of the kind $m_{H} / m_{t}$, but also ratios of $Q / m_{t}$ with $Q$ being an additional hard scale. In this paper we consider effects of such additional large scales induced by the observables, namely the production of single Higgs bosons at large transverse momenta or far off-shell. 
Being produced at large transverse momenta the Higgs boson will recoil against a hard jet system. For additional jets in the single Higgs production process it is well known that the Higgs transverse momentum distribution shows a logarithmic top mass dependence [52, 53]. Recently, this effect has been proposed as a handle to test the structure of the loopinduced coupling and the underlying Standard Model assumption that the Higgs-gluon coupling is exclusively due to heavy quark loops [54-60]. In phase space regions where this logarithmic dependence occurs, the two-jet contribution cannot be neglected [61]. This is because it exhibits the same logarithmic structure as the one-jet contribution and its rate at large transverse Higgs momenta is not suppressed compared to the one-jet rate. This necessitates an accurate description of hard jet radiation beyond the simple parton shower.

An alternative method to probe the loop-induced Higgs-gluon coupling is linked to off-shell production of the Higgs with a subsequent decay into four leptons. Initially, it was noticed that off-shell Higgs production and decay in this channel does not exhibit the usual $\Gamma / m$ suppression [69-72]. The reason for this is that the off-shell suppression of the Higgs propagator is partially compensated by lifting the off-shell suppression of the softer of the $Z$ propagators. The interference with the $Z Z$ background further modifies this profile via a negative contribution. This problem can be turned into a virtue when we add off-shell Higgs production with its modified dependence on the Higgs width to the set of Higgs measurements [73-75]. The initial claim that this defines a 'model independent' measurement of the Higgs width ignores the fact that the Higgs-gluon coupling is induced by loops and hence its momentum dependence only follows once we make an assumption of the particles contributing to this loop [76].

Alternatively, we can relate the width measurement to a determination of the full set of dimension- 6 operators and their coefficients induced by an unspecified new physics scenario [77]. Again, we can make use of a logarithmic top mass dependence, now in the distribution of the momentum flowing through the Higgs propagator [78, 79]. While this interpretation runs into problems with a consistent effective theory description we can ask a slightly different question: can we track the top mass dependence of the loopinduced Higgs-gluon coupling and is there any indication for a more generic dimension- 6 interaction? This is exactly the same question which we ask in boosted Higgs plus jets production, which means that we can directly compare the potential of the two kinematic measurements. Moreover, because we know that the top loop contributes to the effective Higgs-gluon coupling we can link direct measurements of the top Yukawa to the study of the effective Higgs-gluon coupling and compare their respective prospects [8, 9].

To link boosted Higgs production with off-shell Higgs production for this specific physics question we define a theoretical framework. It should allow us to test if the top Yukawa coupling is indeed responsible for the observed Higgs-gluon coupling, or if other particles contribute to the corresponding dimension- 6 operator. The relevant part of the Higgs interaction Lagrangian including a finite top mass and free couplings reads [56, 61]

$$
\begin{array}{rlrl}
\mathcal{L}=\mathcal{L}_{\mathrm{SM}}+ & {\left[\Delta_{t} g_{g g H}+\Delta_{g} \frac{\alpha_{s}}{12 \pi}\right] \frac{H}{v} G \mu \nu G^{\mu \nu}-\Delta_{t} \frac{m_{t}}{v} H\left(\bar{t}_{R} t_{L}+\text { h.c. }\right)} & & \text { SFITTER }[8,9] \\
=\left.\mathcal{L}\right|_{\kappa_{j}=0}+\left[\kappa_{t} g_{g g H}+\kappa_{g} \frac{\alpha_{s}}{12 \pi}\right] \frac{H}{v} G_{\mu \nu} G^{\mu \nu}-\kappa_{t} \frac{m_{t}}{v} H\left(\bar{t}_{R} t_{L}+\text { h.c. }\right) & \text { ref. [56]. }
\end{array}
$$


This Higgs-top Lagrangian will be the basis for the analysis presented in this paper. The SFITTER conventions show how $\kappa_{t}$ as well as $\kappa_{g}$ are directly accessible in LHC coupling analyses. While the effective coupling $g_{g g H}$ retains the full top mass dependence, the dimension6 operator is defined without any reference to the top mass and assuming that the entire momentum dependence arises from the gluon field strengths. We will use the SFitTER interpretation to eventually compare the expected performance of the distribution-based search strategies to the usual Higgs coupling analysis. The Standard Model limit is given by $\Delta_{g}=0=\Delta_{t}$. Our two reference points will be

$$
\left(\kappa_{t}, \kappa_{g}\right)_{\mathrm{SM}}=(1,0) \quad \text { and } \quad\left(\kappa_{t}, \kappa_{g}\right)_{\mathrm{BSM}}=(0.7,0.3) .
$$

In the second point the contributions from a top partner to a good approximation compensate for the reduced top Yukawa in the Higgs-gluon coupling, leaving the observed Higgs cross section at the LHC unchanged. This last condition is crucial to get a realistic estimate of the power of distribution-based Higgs analyses, because a significant deviation of the Higgs production rate in gluon fusion will be experimentally accessible long before boosted or off-shell Higgs analysis will become sensitive.

\section{Top mass effects in Higgs rates}

As long as we limit ourselves to the total cross section of the Standard Model Higgs boson, the heavy top limit or low-energy limit provides an accurate prediction for the total rate. The effective Higgs-gluon coupling is then given by a single coupling value [39-42]

$$
\begin{aligned}
\mathcal{L}_{g g H} \supset g_{g g H} \frac{H}{v} G^{\mu \nu} G_{\mu \nu} \\
\frac{g_{g g H}}{v}=\lim _{\tau \rightarrow \infty} \frac{\alpha_{s}}{8 \pi} \frac{1}{v} \tau[1+(1-\tau) f(\tau)] \\
\text { with } \quad f(\tau) \stackrel{\text { on-shell }}{=}\left(\arcsin \sqrt{\frac{1}{\tau}}\right)^{2} \stackrel{\tau \rightarrow \infty}{=} \frac{1}{\tau}+\frac{1}{3 \tau^{2}}+\mathcal{O}\left(\frac{1}{\tau^{3}}\right),
\end{aligned}
$$

all in terms of $\tau=4 m_{t}^{2} / m_{H}^{2}>1$. The usual scalar three-point function is written in the dimensionless form $f(\tau)=-m_{H}^{2} C\left(m_{H}^{2} ; m_{t}, m_{t}, m_{t}\right) / 2$. In principle, the above Lagrangian describes the interaction of up to four gluons with one Higgs bosons and can be generalized to several Higgs bosons. However, if the vertex involves more than two gluons, the given form of $g_{g g H}$ is only correct for the leading term in $m_{H} / m_{t}$. Sub-leading terms in $m_{H} / m_{t}$ also arise from other tensor structures once more than two gluons are involved. Moreover, for the gluon-gluon-Higgs vertex the effective coupling $g_{g g H}$ only accounts for top mass effects if the Higgs and the gluons are on their mass shells. Beyond the assumption of three on-shell particles $g_{g g H}$ fails to describe the dynamics. For example, applied to differential distributions including additional jets, like the transverse momentum of the Higgs boson in LHC production, the low-energy approximation in the dimension- 6 operator breaks down. In this regime, the top contribution in the loop starts to be resolved and leads to effects in the distributions. Similarly, when we allow the singly produced Higgs boson to be offshell, the effective Higgs-gluon coupling $g_{g g H}$ becomes a non-trivial function of the mass scales involved. 

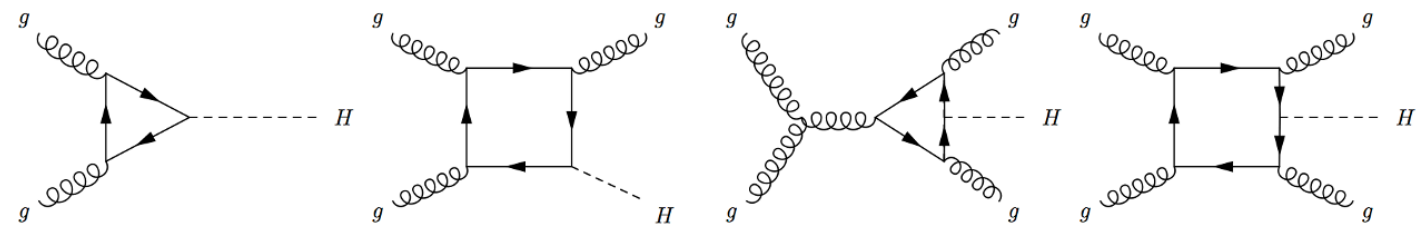

Figure 1. Sample Feynman diagrams contributing for Higgs production with up to 2 jets at leading order.
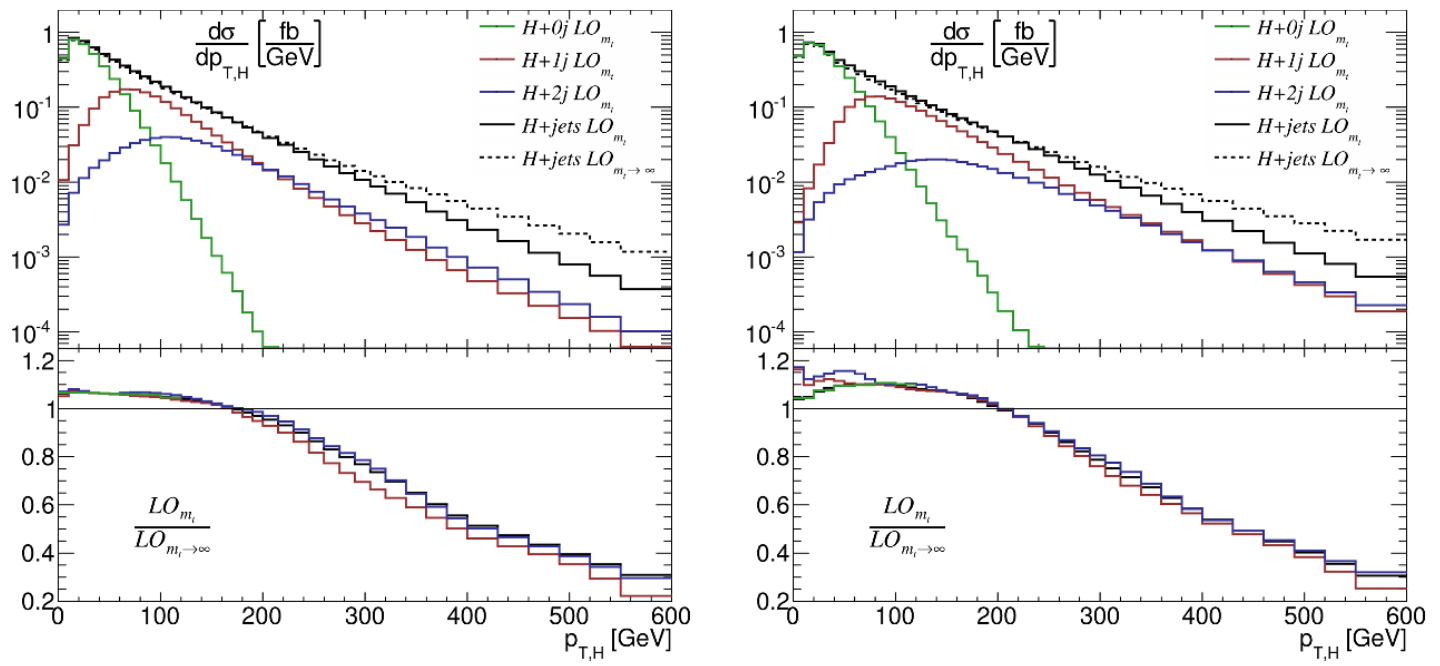

Figure 2. Transverse momentum distribution $p_{T, H}$ for $H \rightarrow W W+$ jets production at LO with Sherpa (left panel) and Pythia8 (right panel). We present the distributions for exclusive and merged jet samples with finite top mass effects $\left(m_{t}=173 \mathrm{GeV}\right)$ and in the low-energy approximation $\left(m_{t} \rightarrow \infty\right)$. We assume the LHC at $\sqrt{S}=13 \mathrm{TeV}$.

In this section we present a state-of-art event simulation including top mass effects beyond the low-energy limit. It relies on the general purpose Monte Carlo event generator SherPa [81-84]. We use SHERPA to generate events for Higgs boson production in association with up to 2 jets and the corresponding backgrounds, $W W$ and top pair production. In all cases we apply multi-jet merging of the matrix elements and the parton showers at LO with the algorithm presented in refs. $[85,86]$ or, where not otherwise stated at NLO according to the MEPs@NLO algorithm [87, 88]. In each case, the implementation is automated once the respective virtual matrix elements are available. This way we generate NLO-merged events for Higgs production with jets in the low-energy limit [89]. In this paper we extend this implementation by top (and bottom) mass effects at leading order accuracy by using loop-level matrix elements provided by OpenLoops [90-93], which we use for reweighting the effective theory.

As a starting point, we study the impact of the top mass corrections in the Higgs boson transverse momentum using LO multi-jet merging with up to two jets. A sample of the representative Feynman diagrams are displayed in figure 1. We assume fully leptonic Higgs decays $H \rightarrow W_{\ell} W_{\ell}+$ jets. In the left panel of figure 2 we display the LO-merged 
results from SherPA. Because this automized implementation relies on the low-energy limit for the Higgs-gluon coupling described in eq. (2.1), we reweight all tree-level matrix elements in the effective theory with their full loop counterparts. This defines a correction factor [94-96]

$$
r_{t}^{(n)}=\frac{\left|\mathcal{M}^{(n)}\left(m_{t}\right)\right|^{2}}{\left|\mathcal{M}^{(n)}\left(m_{t} \rightarrow \infty\right)\right|^{2}}
$$

for each jet multiplicity $n$. In the right panel of figure 2 we also show the corresponding results from Pythia8 [100], based on the CKKW-L merging. The parton level events for the Pythia merging in the 0-jet bin come from MadGraph5 [101], in the 1-jet bin we use MCFM [102, 103], and in the 2-jet bin we use VBFNLO [104].

At the analysis level, jets are defined using the anti-kT algorithm implemented in Fastjet with $\mathrm{R}=0.5$ and we assume basic acceptance cuts

$$
\begin{array}{ll}
p_{T, \ell}>20 \mathrm{GeV} & \left|\eta_{\ell}\right|<2.5 \\
p_{T, j}>30 \mathrm{GeV} & \left|\eta_{j}\right|<4.5 .
\end{array}
$$

The individual curves for the different jet bins account for the number of jets passing these acceptance cuts, rather than the number of hard jets entering the merging procedure. The results from SHERPA and PYTHIA8 broadly agree with each other. In both simulations we observe that for all contributions the low-energy limit and the full results scale the same way as long as $p_{T, H} \lesssim m_{t}$. In this regime the only difference is a constant scaling factor 1.065 for the Higgs-gluon coupling. Although $b$-quark loops become relevant in this regime and need to be accounted, they present sub-leading contributions in the boosted regime. Since we will be mostly concerned with boosted Higgs of $p_{T, H} \gtrsim m_{t}$ these contributions can be safely neglected [107-109].

Above this energy scale the effective and full theory start to visibly diverge. Looking at the jet multiplicities we confirm that this effect is driven by Higgs production with two jets, where the top mass effects are not only larger than in the one-jet process relative to the respective cross section, but also larger in absolute terms [61]. In the lower panels of figure 2 we see that the top mass effects lead to contributions as large as a factor four in the rate at transverse momenta of $600 \mathrm{GeV} .^{1}$ Another remarkable feature which we observe in figure 2 is that the top mass effects factorize: the full top mass dependence provides the same $p_{T, H}$-dependent correction factor for each jet bin, and consequently for the merged result. Thus, the leading contribution could be approximately described by the production of the Higgs boson with one hard jet accompanied by extra jet emissions accounted by the Parton Shower. Notice, however, that the used merging prescription (besides properly capturing these effects, see figure 2) leads to a more precise description of the event rate and kinematics since it also captures the full matrix element information in each jet multiplicity. Finally, the lower panels of figure 2 indicate that an experimental analysis including systematic and theoretical uncertainties can rely on the range $p_{T, H}<m_{t}$ as a safe reference region, searching for a distinct turn-over in the distribution around $p_{T, H}=m_{t}$.

\footnotetext{
${ }^{1}$ The size of these effects suggest that for any strongly boosted Higgs analysis a proper modelling of the top mass effects is of vital importance.
} 
This observed factorization at leading order strengthens the basic assumption underlying our precision study, namely that top mass effects in Higgs production are fully associated with the hard process. This is know to not apply to bottom mass effect, which we assume to be small and not critical for the phase space regions we consider [107-109]. Hence, we can use the SHERPA results in the low-energy limit and reweight them on an event-by-event basis with the corresponding heavy-quark matrix element.

The MEPS@NLO algorithm [87, 88] for multi-jet merging of NLO matrix elements can be viewed, intuitively, as stacking towers of individual MC@NLO simulations [97] on top of each other, without a double counting of emissions. The only subtlety in the SHERPA implementation $[98,99]$ is that the actual implementation of the MC@NLO algorithm has been slightly changed to also include sub-leading color effects in the Sudakov form factor. To see in more detail how this works at NLO, let us consider the structure of the SMC@NLO cross section (including the first emission)

$$
\mathrm{d} \sigma^{\mathrm{S}-\mathrm{MC} @ \mathrm{NLO}}=\mathrm{d} \Phi_{n}\left[\mathcal{B}+\mathcal{V}+\int \mathrm{d} \Phi_{1} \mathcal{D}\right]\left(\Delta\left(t_{0}\right)+\int \mathrm{d} \Phi_{1} \frac{\mathcal{D}}{\mathcal{B}} \Delta(t)\right)+\mathrm{d} \Phi_{n+1}[\mathcal{R}-\mathcal{D}]
$$

where $\mathcal{B}, \mathcal{V}$ and $\mathcal{R}$ denote the Born, virtual and real emission contributions associated with the $n$ and $n+1$ particle phase space integrals. The S-MC@NLO resummation kernel $\mathcal{D}=\widetilde{\mathcal{B}} \otimes \mathcal{K}$ is constructed from a color-correlated and spin-correlated Born matrix element $\widetilde{\mathcal{B}}$ and a suitable splitting function $\mathcal{K}[97,110,111]$. By construction, $\mathcal{D}$ coincides with the real emission matrix element in the soft and/or collinear limit. Note that in S-MC@NLO the ratio $\mathcal{D} / \mathcal{B}$ also constitutes the kernel of the Sudakov form factor for the first emission, in difference to the original MC@NLO method.

In a second step we reweight all tree-level matrix elements in the low-energy limit with their full loop counterparts. This gives rise to correction factors $r_{t}^{(n)}$ defined in eq. (2.2) modifying the merged rate prediction in eq. (2.4),

$\mathrm{d} \sigma^{\mathrm{S}-\mathrm{MC} @ \mathrm{NLO}}=\mathrm{d} \Phi_{n} r_{t}^{(n)}\left[\mathcal{B}+\mathcal{V}+\int \mathrm{d} \Phi_{1} \mathcal{D}\right]\left(\Delta\left(t_{0}\right)+\int \mathrm{d} \Phi_{1} \frac{\mathcal{D}}{\mathcal{B}} \Delta(t)\right)+\mathrm{d} \Phi_{n+1}\left[r_{t}^{(n+1)} \mathcal{R}-r_{t}^{(n)} \mathcal{D}\right]$.

The NLO corrections in the low-energy limit and the top mass corrections are thus applied in a factorized form. This prescription offers a gauge invariant interpolation between both types of corrections. It is worth noting that the resummation properties of the SMc@NLO kernel are not altered, because its argument is a ratio of matrix elements. The infrared safety of the fixed-order correction is guaranteed as long as $r_{t}^{(n+1)} \rightarrow r_{t}^{(n)}$ in the infrared limit. Our approach generalizes the MEPs@NLO method [87, 88], now including next-to-leading order corrections in the low-energy approximation as well as the top mass dependence at leading order for all jet multiplicities considered. Eventually, it needs to be tested once the two-loop multi-scale diagrams can be evaluated over the full phase space.

Following this implementation we upgrade our boosted Higgs analysis in figure 2 to the NLO level. The 0 -jet and 1-jet bins include the NLO corrections, while the 2-jet bin remains at leading order. In figure 3 we display a sample of the Feynman diagrams in the NLO corrections. The upgraded NLO distributions are presented in figure 4. In the left panel we show that apart from the different total rate all top mass features are completely analogous 

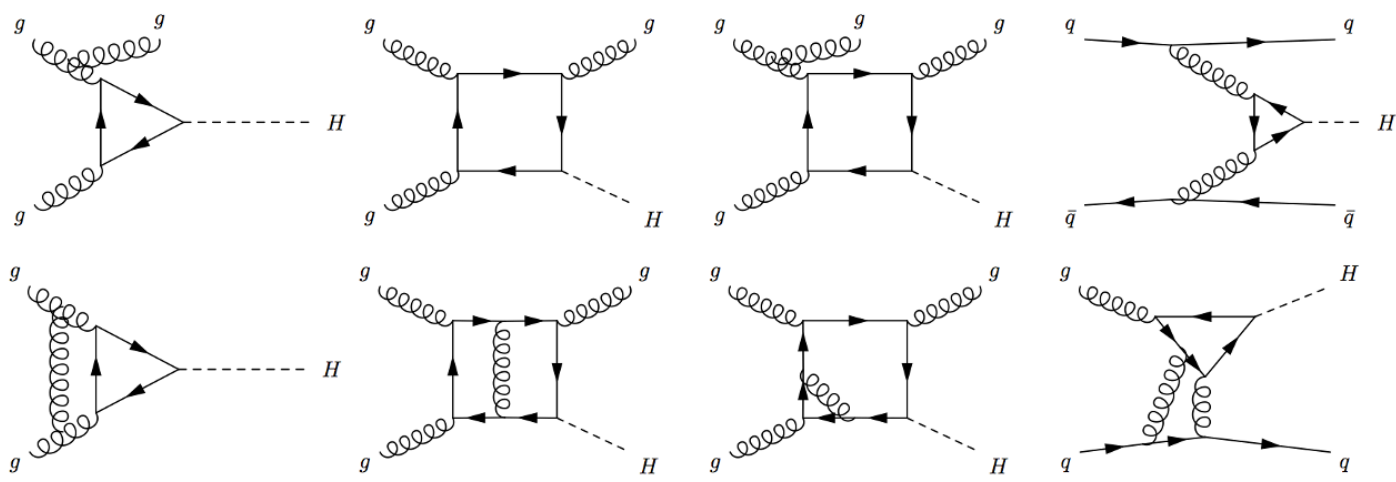

Figure 3. Sample one-loop Feynman diagrams contributing to the Higgs and Higgs-jet production. On the top we display the NLO real corrections and on the bottom the virtual contributions.
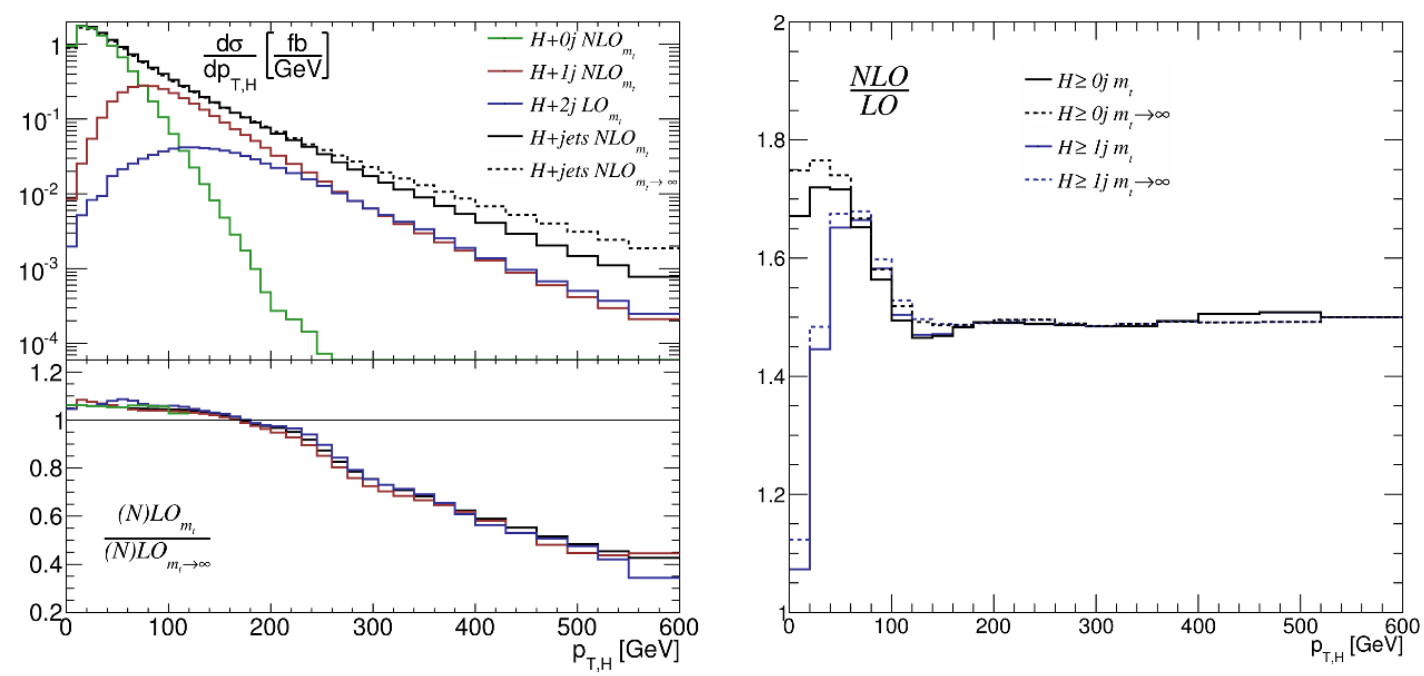

Figure 4. Transverse momentum distribution $p_{T, H}$ for $H \rightarrow W W+$ jets production with SHERPA at NLO (left panel). We present the distributions for exclusive and merged jet samples with finite top mass effects $\left(m_{t}=173 \mathrm{GeV}\right)$ and in the low-energy approximation $\left(m_{t} \rightarrow \infty\right)$. In the right panel we show the $p_{T, H}$-dependent $K$-factor for $H$ and $H+1$ jet production.

to the leading order case. The ratio between the full calculation and low-energy limit shows the same profile. In the right panel, we shown that the NLO corrections factorize, i.e. the relative NLO corrections for the full theory and for the low-energy approximation agree independently for the $H$ and $H+1$ jet rate.

\section{Boosted Higgs production}

For boosted Higgs production the effect of a finite top mass has been known for an eternity: adding jets to the hard process pushes one or two gluon propagators off their respective mass shell. In that case the matrix elements for Higgs production in association with one 
jet [52-54] and two jets [61] develop a top mass dependence

$$
\mathcal{M}_{H j(j)} \propto \frac{m_{t}^{2}}{p_{T, H}}\left(C_{0}+C_{1} \log \frac{p_{T, H}^{2}}{m_{t}^{2}}+C_{2} \log ^{2} \frac{p_{T, H}^{2}}{m_{t}^{2}}\right),
$$

where $C_{0}, C_{1}$ and $C_{2}$ are formed by the combination of constants and $m_{t}$ independent terms. Beyond this $m_{t}$ logarithmic dependence absorptive parts of the one-loop integrals exist, but are unfortunately too small to be observed in the coming LHC run(s) [61]. If we follow eq. (1.1) and allow for a top quark as well as an unspecified heavy state in the loop-induced Higgs-gluon coupling, we can write the matrix element for Higgs production in gluon fusion as

$$
\mathcal{M}=\kappa_{t} \mathcal{M}_{t}+\kappa_{g} \mathcal{M}_{g} .
$$

The index $t$ marks the top contributions, while $g$ the contributions from the dimension- 6 Higgs-gluon operator in the low-energy limit. All prefactors except for the $\kappa_{j}$ are absorbed in the definitions of $\mathcal{M}_{j}$.

Following the bench mark point in eq. (1.2) we will be specially interested in deviations from the Standard Model, where the two couplings satisfy $\kappa_{t}+\kappa_{g}=1$. The transverse momentum distribution will then allow us to disentangle the effects of $\kappa_{t}$ and $\kappa_{g}$ while respecting the experimental constrains on the Higgs production cross section $\sigma \sim\left|\kappa_{t}+\kappa_{g}\right|^{2}$. For a kinematic distribution like the Higgs transverse momentum this means

$$
\frac{d \sigma}{d p_{T, H}}=\kappa_{t}^{2} \frac{d \sigma_{t t}}{d p_{T, H}}+\kappa_{t} \kappa_{g} \frac{d \sigma_{t g}}{d p_{T, H}}+\kappa_{g}^{2} \frac{d \sigma_{g g}}{d p_{T, H}} .
$$

To access the different components in the Higgs-gluon coupling one needs to decouple the soft and hard momentum components flowing in this loop-induced coupling. The separation of these factors can be efficiently achieved through the kinematics of Higgs plus jets production. This feature was studied for the 1-jet or 2-jet cases, indicating that we can achieve a decent sensitivity for ratio for integrated luminosities of $\mathcal{O}\left(1 \mathrm{ab}^{-1}\right)[54-56,61,68]$. Because the logarithmic dependence in eq. (3.1) is the same for the 1-jet and 2-jet hard matrix elements, and because our discussion in section 2 shows that the same holds true even for relatively low $p_{T, H} \gtrsim m_{t}$, we have to use multi-jet merging to provide a reliable estimate of these effects. This also implies that in an optimized combined analysis we should focus on jet-inclusive observables like $p_{T, H}$ to combine different jet multiplicities. As a side effect, the merged approach guarantees a reliable description for the distributions over the full momentum range, as we can see in figures 2 and 4 .

In figure 5 we show two of the key distributions which allow us to separate the Higgs signal from the different background. The azimuthal angle between the two forward jets is a well known probe for the Lorentz structure of the hard process [112-119]. Because it is only defined for at least two additional hard jets, it is one of the key improvements of our merged analysis over Higgs production with a single hard jet. The corresponding background rejection cuts are given in table 1 . The signal events are generated with NLO predictions for the 0-jet and 1-jet processes and LO precision for the hard 2-jet process. The $t \bar{t}+$ jets background includes the NLO prediction for the 0 -jet bin and up to 3 hard 

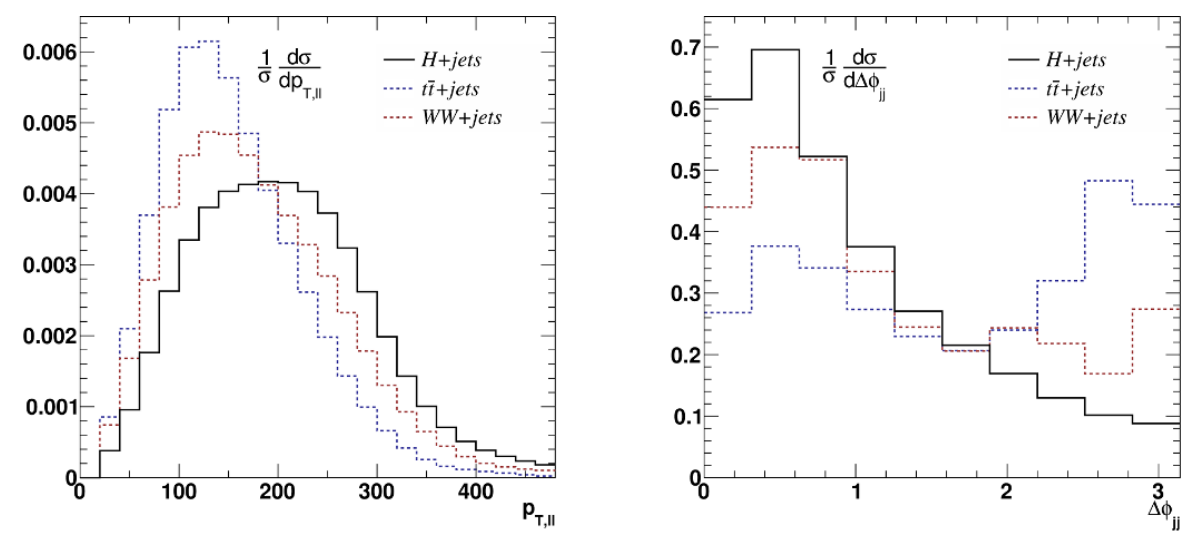

Figure 5. Normalized $p_{T, l l}$ (left) and $\Delta \phi_{j j}$ (right) distributions for the $H \rightarrow W W$ signal and the dominant backgrounds. All universal cuts listed in table 1 are already applied. We assume the LHC at $\sqrt{S}=13 \mathrm{TeV}$.

\begin{tabular}{|c|c|c|c|c|c|c|}
\hline & \multicolumn{3}{|c|}{$(H \rightarrow W W)+(0+1) j$} & \multicolumn{3}{|c|}{$(H \rightarrow W W) j j$ inclusive } \\
\hline cuts & $H+$ jets & $W W+$ jets & $t \bar{t}+$ jets & $H+$ jets & $W W+$ jets & $t \bar{t}+$ jets \\
\hline $\begin{array}{l}p_{T, j}>40 \mathrm{GeV},\left|y_{j}\right|<4.5 \\
p_{T, \ell}>20 \mathrm{GeV},\left|y_{\ell}\right|<2.5\end{array}$ & 87.9 & 3220 & 9640 & 6.50 & 203 & 5890 \\
\hline$N_{b}=0$ & 84.9 & 3180 & 7400 & 5.09 & 189 & 2790 \\
\hline$m_{\ell \ell} \in[10,60] \mathrm{GeV}$ & 69.0 & 628 & 1470 & 4.22 & 36.2 & 503 \\
\hline$\notin_{T}>45 \mathrm{GeV}$ & 49.7 & 504 & 1250 & 3.55 & 32.6 & 493 \\
\hline$\Delta \phi_{\ell \ell}<0.8$ & 24.0 & 195 & 561 & 2.78 & 20.2 & 237 \\
\hline$m_{T}<125 \mathrm{GeV}$ & 23.7 & 74.5 & 250 & 2.75 & 10.8 & 119 \\
\hline$p_{T, H}>300 \mathrm{GeV}$ & 0.27 & 1.41 & 1.24 & 0.42 & 2.12 & 5.32 \\
\hline$p_{T, l l}>180 \mathrm{GeV}$ & 0.15 & 0.58 & 0.35 & 0.24 & 0.98 & 1.87 \\
\hline$\Delta \phi_{j j}<1.8$ & & & & 0.21 & 0.69 & 0.90 \\
\hline
\end{tabular}

Table 1. Cut flow for $H+$ jets, $W W+$ jets and $t \bar{t}+$ jets. All events are generated with Sherpa using the MEPS@NLO algorithm. The rates are given in $\mathrm{fb}$.

jets at the LO level. The QCD component from $W W+$ jets production is loop induced and it is generated with up to 1 -jet with LO precision. The electroweak $W W+$ jets component includes NLO corrections up to the 1-jet bin and up to 3 hard jets at LO. The analysis largely correspond to the $W W$ analysis proposed for the 2-jet channel in ref. [61].

In particular when we link different experimental measurements to the same Lagrangian interpretation given by eq. (1.1) the question arises how the experimental approaches compare. In figure 6 we show an idealized projection of the reach in the boosted Higgs analyses. Based on a 2-dimensional $\mathrm{CL}_{s}$ analysis of the number of jets and the transverse momentum of the Higgs, $\left(n_{\text {jets }}, p_{T, H}\right)$, we estimate how much luminosity would be required to rule out our BSM reference point given in eq. (1.2). We find that in the absence of systematic and theoretical uncertainties it would take around $700 \mathrm{fb}^{-1}$ of data 


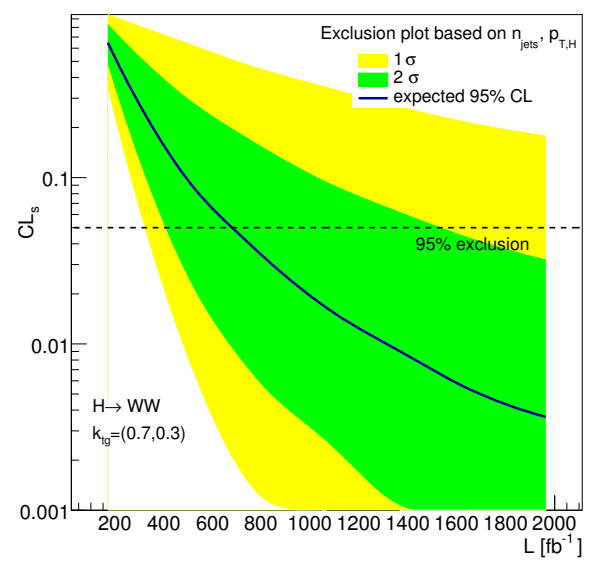

Figure 6. Confidence level for separating the BSM hypotheses $\kappa_{t, g}=(0.7,0.3)$ from the Standard Model. We show results for $H \rightarrow W W$ decays based on the $2 \mathrm{D}$ distribution $\left(n_{\text {jets }}, p_{T, H}\right)$.

to achieve a $95 \%$ C.L. exclusion. Even if we rely on the reference region at $p_{T, H} \lesssim m_{t}$ to efficiently reduce the uncertainties, a meaningful study of boosted Higgs production might require attobarn integrated luminosities. On that time scale it appears unlikely that such a detailed kinematic analysis will be able to compete with a dedicated hypothesis test based on Higgs couplings and including $t \bar{t} H$ production with the combined Higgs decays $H \rightarrow b \bar{b}, \tau \tau, \gamma \gamma[8,9,25-31]$.

\section{Off-shell Higgs production}

One of novel LHC measurement in 2014 is the Higgs width limit from off-shell Higgs production [69-71, 73-75]. For example, CMS published the first results on a rate measurement of $p p \rightarrow Z^{(*)} Z^{(*)} \rightarrow 4$ leptons at high invariant mass $m_{4 \ell}$ [73-75]. This process relies on off-shell contributions from $s$-channel Higgs production. This way, it carries information on the Higgs couplings at different energy scales which could, similarly to the boosted case, probe the energy dependence of the higher-dimensional operators. In this spirit, we exploit the off-shell Higgs regime of $Z Z$ production to probe the Higgs-top sector of the Standard Model [79, 80].

The Higgs contribution to $Z$-pair production is generated via gluon fusion through heavy quark loops. It faces two dominant backgrounds: $q \bar{q} \rightarrow Z Z$ and $g g \rightarrow Z Z$. The $q \bar{q}$ component is generated already at the tree level and constitutes the most important contribution. It is approximately one order of magnitude larger than the gluon fusion part. On the other hand, the gluon fusion contribution features an interference with the Higgs signal in the off-shell $m_{Z Z}$ regime. In figure 7 we display a sample of the contributing Feynman diagrams to $Z Z$ production.

At high invariant mass $m_{Z Z}$ the Higgs decays mostly into longitudinal gauge bosons. This means that the signal amplitude can be understood from the longitudinal components [78-80]

$$
\mathcal{M}_{t}^{++00}=-2 \frac{m_{4 \ell}^{2}-2 m_{Z}^{2}}{m_{Z}^{2}} \frac{m_{t}^{2}}{m_{4 \ell}^{2}-m_{H}^{2}+i \Gamma_{H} m_{H}}\left[1+\left(1-\frac{4 m_{t}^{2}}{m_{4 \ell}^{2}}\right) f\left(\frac{4 m_{t}^{2}}{m_{4 \ell}^{2}}\right)\right],
$$



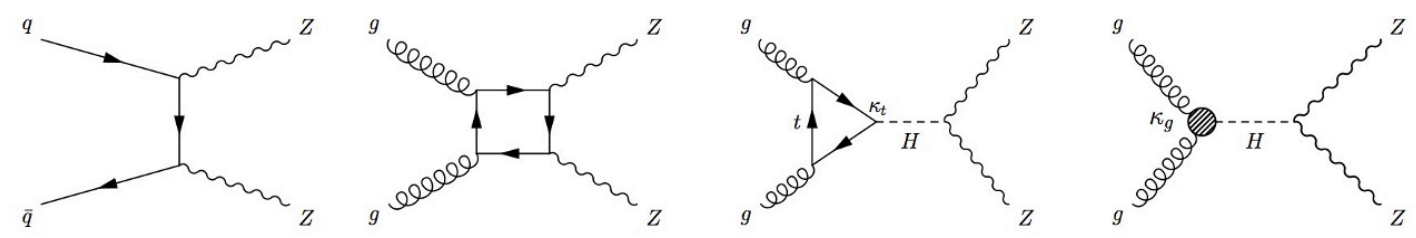

Figure 7. Sample Feynman diagrams for the continuum background $q \bar{q}(g g) \rightarrow Z Z$ (left) and for the signal $g g \rightarrow H \rightarrow Z Z$ (right) with full top mass dependence and in the $m_{t} \rightarrow \infty$ approximation.

where $\Gamma_{H}$ is the Higgs boson width and $f=-m_{4 \ell}^{2} C\left(m_{4 \ell}^{2} ; m_{t}, m_{t}, m_{t}\right) / 2$ represents the dimensionless scalar three-point function. This form corresponds to the on-shell case in eq. (2.1), replacing $\tau=4 m_{t}^{2} / m_{H}^{2}$ with its off-shell analogue $4 m_{t}^{2} / m_{4 \ell}^{2}$ and relying on the form

$$
f(\tau)=-\frac{1}{4}\left(\log \frac{1+\sqrt{1-\tau}}{1-\sqrt{1-\tau}}-i \pi\right)^{2}
$$

for the scalar integral with $\tau<1$. In the low-energy limit far above the Higgs mass shell, $m_{t} \gg m_{4 \ell} \gg m_{H}, m_{Z}$, the scalar integral scales like $f \sim m_{4 \ell}^{2} /\left(4 m_{t}^{2}\right)$ and gives the usual finite effective Higgs-gluon coupling $g_{g g H}$ defined in eq. (2.1). Obviously, this assumption is not correct once we include the actual mass values. Instead, for $\mathcal{M}_{t}$ we better assume $m_{4 \ell} \gg m_{t} \gtrsim m_{H}, m_{Z}$, giving us

$$
\begin{array}{rlrl}
\mathcal{M}_{g}^{++00} & \approx-\frac{m_{4 \ell}^{2}}{2 m_{Z}^{2}} & \text { with } & m_{t} \gg m_{4 \ell} \gg m_{H}, m_{Z} \\
\mathcal{M}_{t}^{++00} \approx+\frac{m_{t}^{2}}{2 m_{Z}^{2}} \log ^{2} \frac{m_{4 \ell}^{2}}{m_{t}^{2}} & \text { with } & m_{4 \ell} \gg m_{t} \gtrsim m_{H}, m_{Z} \\
\mathcal{M}_{c}^{++00} \approx-\frac{m_{t}^{2}}{2 m_{Z}^{2}} \log ^{2} \frac{m_{4 \ell}^{2}}{m_{t}^{2}} & \text { with } & m_{4 \ell} \gg m_{t} \gtrsim m_{Z} .
\end{array}
$$

In the proper limit a logarithmic dependence on $m_{4 \ell} / m_{t}$ develops far above the Higgs mass shell. It is very similar to the transverse momentum dependence in the boosted regime, as seen in eq. (3.1). The ultraviolet logarithm cancels between the correct Higgs amplitude and the continuum, ensuring the proper ultraviolet behavior of the full amplitude. Most importantly, there appears a sign difference between the full top mass dependence and the low-energy limit. For the interference pattern with the continuum process $g g \rightarrow Z Z$ the full top mass dependence predicts a destructive interference whereas in the low-energy limit the interference far above mass shell should be constructive.

Following the parametrization in eqs. (1.1) and (3.2) and including the interference with the continuum background arising from the box diagrams, we can write the gluoninduced amplitude $g g \rightarrow Z Z$ as

$$
\mathcal{M}_{Z Z}=\kappa_{t} \mathcal{M}_{t}+\kappa_{g} \mathcal{M}_{g}+\mathcal{M}_{c}
$$

Correspondingly, the differential cross section can be expressed as

$$
\frac{d \sigma}{d m_{4 \ell}}=\frac{d \sigma_{c}}{d m_{4 \ell}}+\kappa_{t} \frac{d \sigma_{t c}}{d m_{4 \ell}}+\kappa_{g} \frac{d \sigma_{g c}}{d m_{4 \ell}}+\kappa_{t}^{2} \frac{d \sigma_{t t}}{d m_{4 \ell}}+\kappa_{t} \kappa_{g} \frac{d \sigma_{t g}}{d m_{4 \ell}}+\kappa_{g}^{2} \frac{d \sigma_{g g}}{d m_{4 \ell}} .
$$



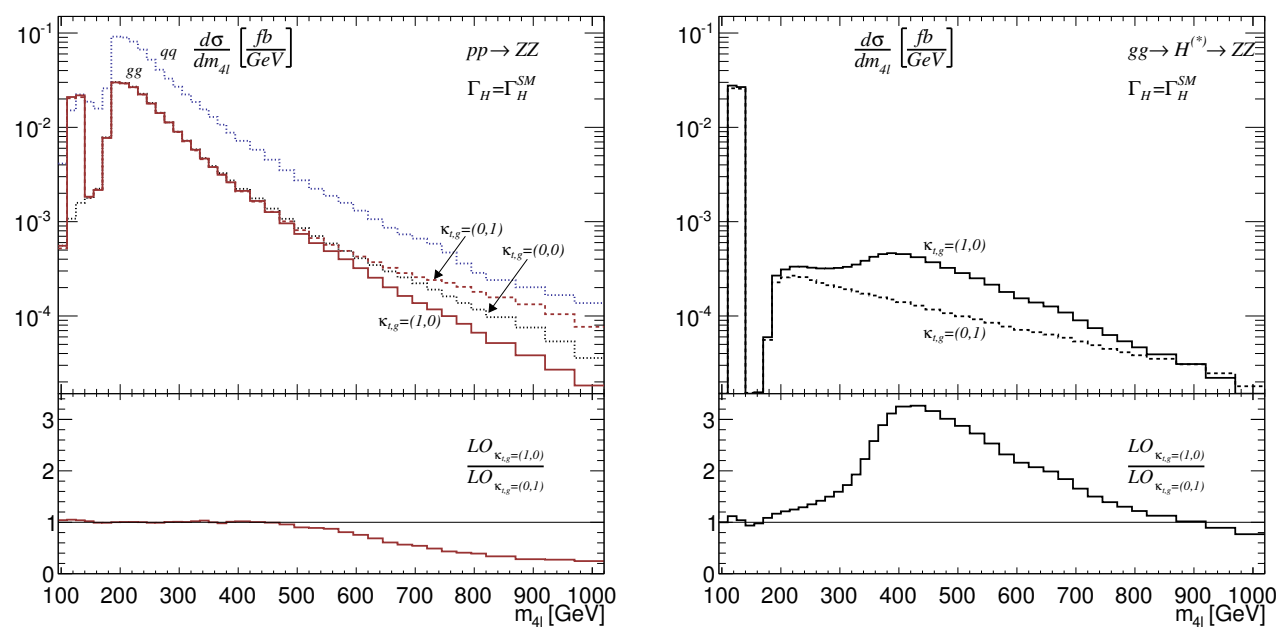

Figure 8. $m_{4 l}$ distributions for the $q \bar{q}(g g) \rightarrow Z Z$ (left panel) and $g g \rightarrow H^{(*)} \rightarrow Z Z$ (right panel) for the different signal hypothesis and the dominant background. We assume the LHC at $\sqrt{S}=13 \mathrm{TeV}$.

Using this parametrization, we can access each of the different components by switching on and off the coefficients $\kappa_{t}$ and $\kappa_{g}$.

The gluon-initiated and quark-initiated $p p \rightarrow e^{+} e^{-} \mu^{+} \mu^{-}$signal and background events are generated with MCFM-6.8 [102, 103], respectively at LO and NLO. We modify the original MCFM code to separately access all components defined in eq. (4.5). All our results follow the CMS cut-flow analysis [73-75]

$$
\begin{aligned}
p_{T, \mu} & >5 \mathrm{GeV} & & \left|\eta_{\mu}\right|<2.4 \\
p_{T, e} & >7 \mathrm{GeV} & & \left|\eta_{e}\right|<2.5 \\
m_{\ell \ell^{\prime}} & >4 \mathrm{GeV} & & m_{4 \ell}>100 \mathrm{GeV} .
\end{aligned}
$$

For the decay leptons we require transverse momenta above 20 (10) GeV for the leading (sub-leading) lepton and invariant masses of $40<m_{\ell \ell}<120 \mathrm{GeV}\left(12<m_{\ell \ell}<120 \mathrm{GeV}\right)$ for the leading (sub-leading) same-flavor lepton pair. We take the renormalization and factorization scales to be $m_{4 \ell} / 2$ and use the PDF set CTEQ6L1 [120].

The Higgs production process $g g \rightarrow H^{(*)} \rightarrow Z Z$ has been computed to very high precision. This includes NNLO and NNLL contributions for inclusive production and at NNLO for the $m_{Z Z}$ distribution [121]. On the other hand, the continuum production $g g \rightarrow Z Z$ is only known to leading order. However, it was recently demonstrated that the NLO and NNLO effects for the signal and the signal-background interference contributions have a very similar perturbative QCD enhancement [122]. Therefore, we could include these QCD effects by assuming $K$-factors for the signal, signal-background interference and background contributions. In this study we include a differential NLO $K$-factor only.

In figure 8 we present the distributions for the different signal hypotheses defined in eq. (4.4) along with the quark-induced background. In the bottom panel we show the ratio between the top mass dependent coupling $\kappa_{t, g}=(1,0)$ and the low-energy form $\kappa_{t, g}=(0,1)$. As expected from eq. (4.3), in the full process $g g \rightarrow Z Z$ we observe an 
enhancement in the tail for the low-energy limit and a suppression for the full top mass result. In the right panel we focus on Higgs production $g g \rightarrow H^{(*)} \rightarrow Z Z$ and neglect the dominant continuum interference. For this process we observe three threshold effects which largely control the $m_{4 \ell}$ distribution profile: first, we see the on-shell Higgs production peak around $m_{H} \approx 125 \mathrm{GeV}$; second, there is a threshold at $2 m_{Z} \approx 180 \mathrm{GeV}$ which leads to an abrupt enhancement in the cross section without which the off-shell measurements would not be possible [73-75]; third, a peak appears when both tops in the loop-induced coupling are simultaneously on-shell, $2 m_{t} \approx 350 \mathrm{GeV}$. This last threshold is only present when we include the full top mass dependence. This leads to a clear enhancement from the absorptive top threshold with respect to the effective coupling approach, tantamount to a maximum correction factor of $\mathcal{O}(3)$ shown in the bottom-right panel of figure 8 .

While there are no spin correlations between production and decay in the Higgs amplitudes $\left|\mathcal{M}_{t, g}\right|^{2}$, they appear through the interference with the continuum background $\mathcal{M}_{c}$. This way, the Cabibbo-Maksymowicz-Dell'Aquila-Nelson angles [123-126] help to extract top mass effects and allow us to further suppress the background in the off-shell regime. The set of five angles completely capture the kinematics of the decay $H \rightarrow Z Z \rightarrow 4 \ell$, including the correlation with the incoming protons. The momenta from the Higgs decay are given by

$$
p_{H}=p_{Z_{e}}+p_{Z_{\mu}} \quad p_{Z_{e}}=p_{e^{-}}+p_{e^{+}} \quad p_{Z_{\mu}}=p_{\mu^{-}}+p_{\mu^{+}} .
$$

For each of these momenta and the beam direction we define unit three-momenta $\hat{p}_{i}$ in the Higgs rest frame and in the two $Z_{e, \mu}$ rest frames

$$
\begin{aligned}
\cos \theta_{e} & =\left.\hat{p}_{e^{-}} \cdot \hat{p}_{Z_{\mu}}\right|_{Z_{e}} & \cos \theta_{\mu} & =\left.\hat{p}_{\mu^{-}} \cdot \hat{p}_{Z_{e}}\right|_{Z_{\mu}} \quad \cos \theta^{*}=\left.\hat{p}_{Z_{e}} \cdot \hat{p}_{\text {beam }}\right|_{H} \\
\cos \phi_{e} & =\left.\left(\hat{p}_{\text {beam }} \times \hat{p}_{Z_{\mu}}\right) \cdot\left(\hat{p}_{Z_{\mu}} \times \hat{p}_{e^{-}}\right)\right|_{Z_{e}} & \cos \Delta \phi & =\left.\left(\hat{p}_{e^{-}} \times \hat{p}_{e^{+}}\right) \cdot\left(\hat{p}_{\mu^{-}} \times \hat{p}_{\mu^{+}}\right)\right|_{H} \cdot
\end{aligned}
$$

In figure 9 we present the the normalized angular distributions. Notice that the main sensitivity at high $m_{4 \ell}$ appears in the two polarization angles $\cos \theta_{\ell}$.

To roughly compare the potential of the boosted Higgs production and off-shell Higgs production in probing the effective Higgs-gluon coupling we analyze the information from the different $m_{4 \ell}$ bins using the $\mathrm{CL}_{s}$ method. To enhance the signal sensitivity we use the angular correlations in two ways. First, we suppress the $q \bar{q} \rightarrow Z Z$ background by requiring that $\left|\cos \Theta^{*}\right|<0.7$. Second, we include the leading angle in a 2 -dimensional $\mathrm{CL}_{s}$ analysis of $\left(\cos \theta_{e}, m_{4 \ell}\right)$. In figure 10 we see that even using an overly optimistic setup, where we neglect systematic as well as theory uncertainties, the off-shell channel will only allow for an exclusion of our BSM hypothesis defined in eq. (1.2) based on a few inverse attobarns of integrated luminosity. Compared to the reach of the idealized results from boosted Higgs production shown in figure 6 this is not promising. Moreover, measuring the top Yukawa using $t \bar{t} H$ production and the combined Higgs decays $H \rightarrow b \bar{b}, \tau \tau, \gamma \gamma$ will easily constrain a modified structure of the Higgs-gluon coupling beyond this level $[8,9]$. 

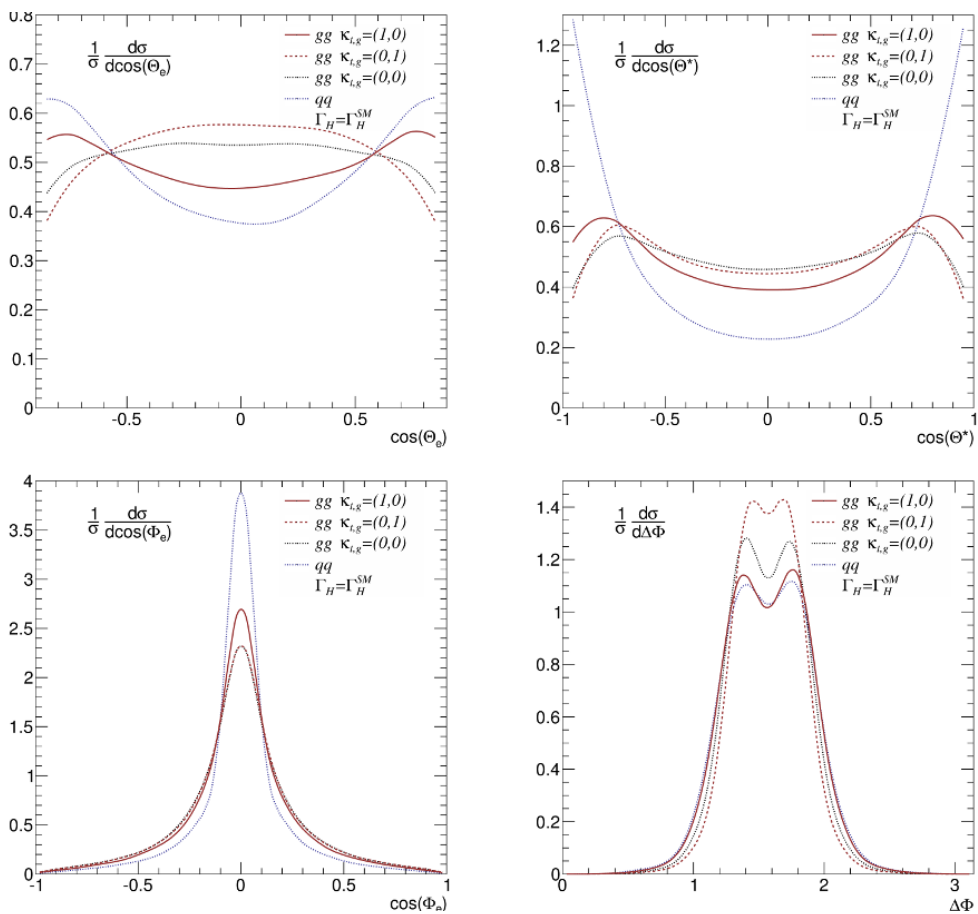

Figure 9. Normalized distributions for the angular correlations in the $g g \rightarrow Z Z$ assuming different signal hypothesis $\kappa_{t, g}$. We also show the quark-induced background $q \bar{q} \rightarrow Z Z$ for $m_{4 \ell}>600 \mathrm{GeV}$. We assume the LHC at $\sqrt{S}=13 \mathrm{TeV}$.

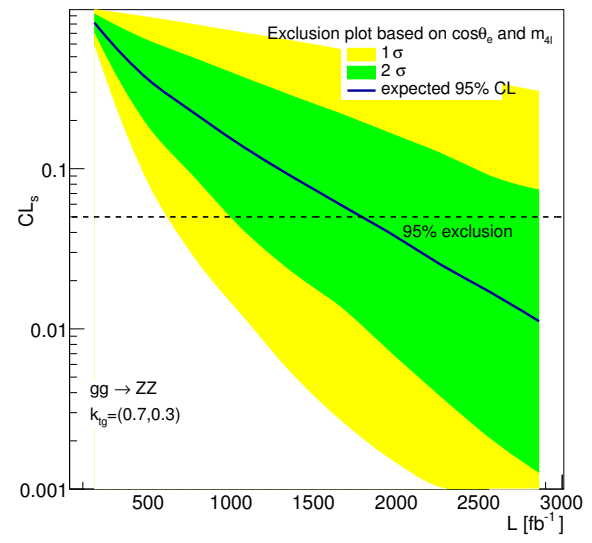

Figure 10. Confidence level for separating the BSM hypotheses $\kappa_{t, g}=(0.7,0.3)$ from the Standard Model. We show results for $g g \rightarrow Z Z$ decays based on the $2 \mathrm{D}$ distribution $\left(\cos \theta_{e}, m_{4 \ell}\right)$.

\section{$5 \quad$ Width measurement}

If combined with on-shell rate measurements, the additional off-shell rate measurements described in section 4 can be used to test the assumptions on the recently proposed Higgs width measurement [69-71, 73-75]. Fundamentally, this proposal aims to break the degen- 
eracy present on the on-shell Higgs couplings analysis, namely

$$
\underset{i \rightarrow H \rightarrow f}{\sigma_{i n-s h e l l}} \propto \frac{g_{i}^{2}\left(m_{H}\right) g_{f}^{2}\left(m_{H}\right)}{\Gamma_{H}}=\text { constant for } \quad g_{i, f}\left(m_{H}\right) \rightarrow \xi g_{i, f}^{\mathrm{SM}}\left(m_{H}\right) \quad \Gamma_{H} \rightarrow \xi^{4} \Gamma_{H}
$$

For example in the SFITTER approach this degeneracy is broken through the assumption that the total Higgs width be the sum of all observed particle Higgs widths [8, 9]. Alternatively, in HiggsSignals [17] the unitarization of the $W W$ scattering amplitude is turned into an upper limit on the Higgs couplings to break this degeneracy. Using offshell rates we exploit the subleading dependence on the Higgs width $\Gamma_{H}$ in the off-shell regime, $\sigma_{i \rightarrow H^{*} \rightarrow f}^{\text {off-shell }} \propto g_{i}^{2}(\sqrt{\hat{s}}) g_{f}^{2}(\sqrt{\hat{s}})$. While it is an interesting idea, it is clearly not a model independent width measurement [76]. The model dependence immediately follows from eqs. (2.1) and (4.1), which reflect the non-trivial momentum dependence of the loopinduced Higgs-gluon coupling. For tree-level Higgs couplings we could assume a weak, well-defined logarithmic running, while for such a loop-induced couplings the momentum structure can be anything. For instance, in our framework the $\kappa_{t}$ and $\kappa_{g}$ corrections to the on-shell rate factorize. In contrast, in the off-shell regime we obtain a non-trivial dependence,

$$
\begin{aligned}
\sigma_{g g \rightarrow H \rightarrow Z Z}^{\text {on-shell }} & \propto\left(\kappa_{t}+\kappa_{g}\right)^{2} \frac{g_{g g H}^{2}\left(m_{H}\right) g_{H Z Z}^{2}\left(m_{H}\right)}{\Gamma_{H}} \\
\sigma_{g g \rightarrow H^{*} \rightarrow Z Z}^{\text {off-shell }} & \propto\left(\kappa_{t} g_{g g H}\left(m_{4 \ell}\right)+\kappa_{g} g_{g g H}\left(m_{H}\right)\right)^{2} g_{H Z Z}^{2}\left(m_{4 \ell}\right) .
\end{aligned}
$$

If we assume a scaling factor $\xi^{4}=25$, the Higgs width will vary by $\Gamma=25 \Gamma_{H}$ while the on-shell signal strength will still be at $\mu^{\text {on-shell }}=1$. In figure 11 we display the outcome of this parameter changing. We observe that the on-shell peak still has the same size as the $\mathrm{SM}$ one, as expected from eq. (5.2). However, the interference between the Higgs diagram $g g \rightarrow H^{*} \rightarrow Z Z$ and the continuum process $g g \rightarrow Z Z$ shows a very different behavior as a function of $m_{4 \ell}$, also shown in eq. (5.2).

This comparison of a top-induced Higgs-gluon coupling with the full top mass dependence and in the low-energy limit might be the easiest way of observing the limitation of model independent Higgs width measurements at the LHC. We need to assume that the quantum structure of the effective top-Higgs coupling is exactly as in the Standard Model $\kappa_{t, g}=(1,0)$ to interpret the current off-shell rate measurement as a measurement of the Higgs width. Additional states increasing the Higgs width cannot contribute to the Higgs-gluon coupling without significantly changing the relation between on-shell and off-shell rates.

\section{Summary}

Top mass effects in Higgs production in gluon fusion can be linked to experimentally relevant changes in some of the fundamental distributions, namely the Higgs transverse momentum and reconstructed mass of the Higgs decay products. We have introduced a state-of-the-art simulation tool based on next-to-leading order multi-jet merging. Our results show that the top mass effects factorize for each number of hard jets to leading 

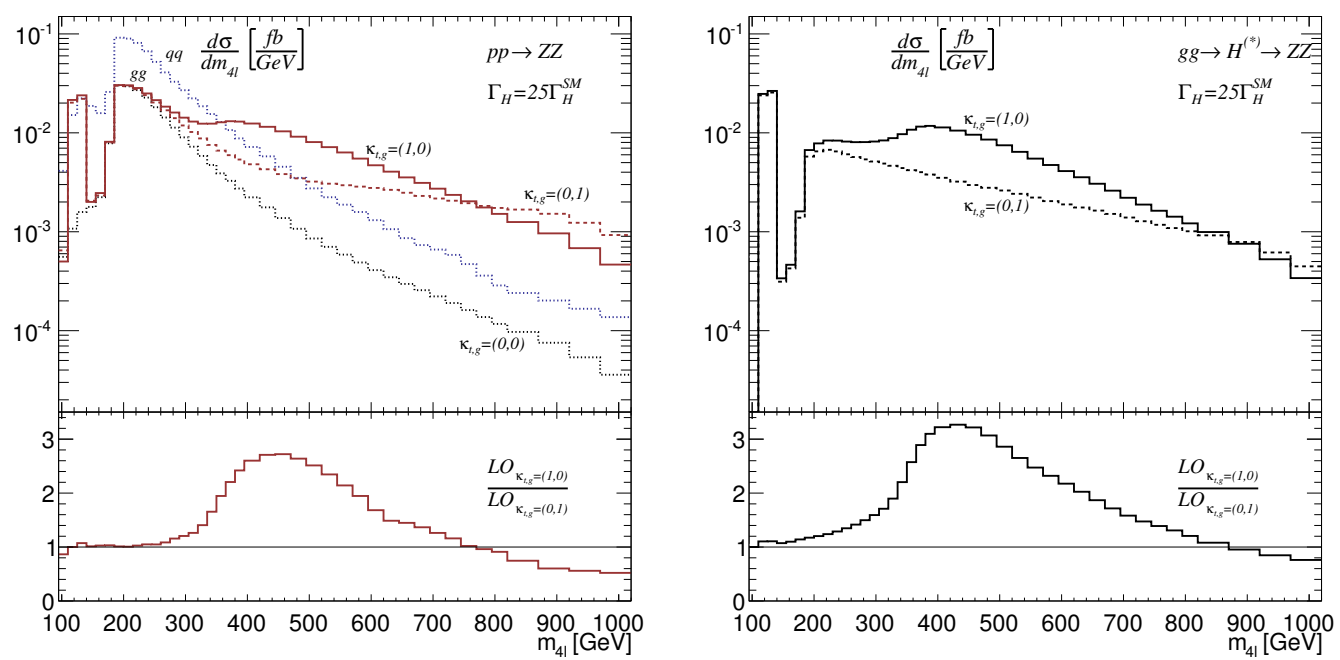

Figure 11. $m_{4 l}$ distributions for the $q \bar{q}(g g) \rightarrow Z Z$ (left panel) and $g g \rightarrow H^{(*)} \rightarrow Z Z$ (right panel) for the different signal hypothesis and the dominant background. For illustration purposes we assume an increased Higgs $\Gamma_{H}=25 \Gamma_{\mathrm{SM}}$.

order and next-to-leading order. This approach allows us to combine Higgs production in association with one and with two hard jets to optimally probe the structure of the Higgs-gluon coupling. Once we include two jets an experimental analysis based on Higgs decays to $W W$ pairs might well be the most promising search channel.

Off-shell production and boosted Higgs production can be interpreted in the same physics framework. While keeping the total Higgs production rate constant we can vary the top Yukawa coupling and an additional dimension-6 Higgs-gluon coupling and search for deviations in key distributions. For a benchmark point with a $30 \%$ reduction of the top Yukawa coupling compensated by a dimension- 6 contribution from physics beyond the Standard Model we estimate the required luminosity for a 95\% C.L. exclusion in both channels. In the absence of systematic and theory uncertainties the boosted Higgs channel will require luminosities close to $1 \mathrm{ab}^{-1}$. Off-shell Higgs production will only rule out the same benchmark value with close to $2 \mathrm{ab}^{-1}$ of integrated luminosity. These numbers can be compared to a standard Higgs coupling analysis: including a conservative estimate of systematic and theoretical uncertainties a coupling extraction of 7 independent Higgs couplings can measure the top Yukawa with a precision of $12 \%$ based on $3 \mathrm{ab}^{-1}$ of LHC data [127].

Finally, we study the top mass dependence of the Higgs-gluon coupling and its impact on the proposed direct Higgs width measurement. It turns out that the width measurement essentially requires the knowledge of the quantum structure of the Higgs-gluon coupling. Additional states responsible for unobserved Higgs decays cannot be linked to this loopinduced interaction.

Open Access. This article is distributed under the terms of the Creative Commons Attribution License (CC-BY 4.0), which permits any use, distribution and reproduction in any medium, provided the original author(s) and source are credited. 


\section{References}

[1] P.W. Higgs, Broken symmetries, massless particles and gauge fields, Phys. Lett. 12 (1964) 132 [INSPIRE].

[2] P.W. Higgs, Broken Symmetries and the Masses of Gauge Bosons, Phys. Rev. Lett. 13 (1964) 508 [INSPIRE].

[3] P.W. Higgs, Spontaneous Symmetry Breakdown without Massless Bosons, Phys. Rev. 145 (1966) 1156 [INSPIRE].

[4] F. Englert and R. Brout, Broken Symmetry and the Mass of Gauge Vector Mesons, Phys. Rev. Lett. 13 (1964) 321 [INSPIRE].

[5] G.S. Guralnik, C.R. Hagen and T.W.B. Kibble, Global Conservation Laws and Massless Particles, Phys. Rev. Lett. 13 (1964) 585 [InSPIRE].

[6] ATLAS collaboration, Observation of a new particle in the search for the Standard Model Higgs boson with the ATLAS detector at the LHC, Phys. Lett. B 716 (2012) 1 [arXiv:1207.7214] [INSPIRE].

[7] CMS collaboration, Observation of a new boson at a mass of $125 \mathrm{GeV}$ with the CMS experiment at the LHC, Phys. Lett. B 716 (2012) 30 [arXiv:1207.7235] [INSPIRE].

[8] M. Klute, R. Lafaye, T. Plehn, M. Rauch and D. Zerwas, Measuring Higgs Couplings from LHC Data, Phys. Rev. Lett. 109 (2012) 101801 [arXiv:1205.2699] [INSPIRE].

[9] D. López-Val, T. Plehn and M. Rauch, Measuring Extended Higgs Sectors as a Consistent Free Couplings Model, JHEP 10 (2013) 134 [arXiv:1308.1979] [INSPIRE].

[10] ATLAS collaboration, An update of combined measurements of the new Higgs-like boson with high mass resolution channels, ATLAS-CONF-2012-170 (2012).

[11] CMS collaboration, Combination of standard model Higgs boson searches and measurements of the properties of the new boson with a mass near $125 \mathrm{GeV}$, CMS-PAS-HIG-12-045.

[12] A. Azatov, R. Contino and J. Galloway, Model-Independent Bounds on a Light Higgs, JHEP 04 (2012) 127 [Erratum ibid. 1304 (2013) 140] [arXiv: 1202.3415] [INSPIRE].

[13] P.P. Giardino, K. Kannike, M. Raidal and A. Strumia, Is the resonance at 125 GeV the Higgs boson?, Phys. Lett. B 718 (2012) 469 [arXiv:1207.1347] [INSPIRE].

[14] J.R. Espinosa, C. Grojean, M. Muhlleitner and M. Trott, First Glimpses at Higgs' face, JHEP 12 (2012) 045 [arXiv: 1207.1717] [INSPIRE].

[15] A. Djouadi and G. Moreau, The couplings of the Higgs boson and its CP properties from fits of the signal strengths and their ratios at the $7+8$ TeV LHC, Eur. Phys. J. C 73 (2013) 2512 [arXiv: 1303.6591] [INSPIRE].

[16] J. Ellis and T. You, Updated Global Analysis of Higgs Couplings, JHEP 06 (2013) 103 [arXiv: 1303.3879] [INSPIRE].

[17] P. Bechtle, S. Heinemeyer, O. Stål, T. Stefaniak and G. Weiglein, HiggsSignals: Confronting arbitrary Higgs sectors with measurements at the Tevatron and the LHC, Eur. Phys. J. C 74 (2014) 2711 [arXiv: 1305.1933] [INSPIRE].

[18] A. Azatov and J. Galloway, Electroweak Symmetry Breaking and the Higgs Boson: Confronting Theories at Colliders, Int. J. Mod. Phys. A 28 (2013) 1330004 [arXiv:1212.1380] [INSPIRE]. 
[19] I. Brivio et al., Disentangling a dynamical Higgs, JHEP 03 (2014) 024 [arXiv:1311.1823] [INSPIRE].

[20] R. Contino, M. Ghezzi, C. Grojean, M. Muhlleitner and M. Spira, Effective Lagrangian for a light Higgs-like scalar, JHEP 07 (2013) 035 [arXiv:1303.3876] [INSPIRE].

[21] J. Elias-Miro, J.R. Espinosa, E. Masso and A. Pomarol, Higgs windows to new physics through $D=6$ operators: constraints and one-loop anomalous dimensions, JHEP 11 (2013) 066 [arXiv: 1308.1879] [INSPIRE].

[22] C. Englert et al., Precision Measurements of Higgs Couplings: Implications for New Physics Scales, J. Phys. G 41 (2014) 113001 [arXiv:1403.7191] [InSPIRE].

[23] J. Ellis, V. Sanz and T. You, Complete Higgs Sector Constraints on Dimension-6 Operators, JHEP 07 (2014) 036 [arXiv: 1404.3667] [InSPIRE].

[24] S. Dawson, I.M. Lewis and M. Zeng, Effective field theory for Higgs boson plus jet production, Phys. Rev. D 90 (2014) 093007 [arXiv:1409.6299] [INSPIRE].

[25] A. Belyaev and L. Reina, $p p \rightarrow t \bar{t} H, H \rightarrow \tau^{+} \tau^{-}$: Toward a model independent determination of the Higgs boson couplings at the LHC, JHEP 08 (2002) 041 [hep-ph/0205270] [INSPIRE].

[26] E. Gross and L. Zivkovic, $t \bar{t} H \rightarrow t \bar{t} \tau^{+} \tau^{-}$: Toward the Measurement of the top-Yukawa Coupling, Eur. Phys. J. C 59 (2009) 731 [INSPIRE].

[27] T. Plehn, G.P. Salam and M. Spannowsky, Fat Jets for a Light Higgs, Phys. Rev. Lett. 104 (2010) 111801 [arXiv:0910.5472] [INSPIRE].

[28] C. Boddy, S. Farrington and C. Hays, Higgs boson coupling sensitivity at the LHC using H-¿̇tau tau decays, Phys. Rev. D 86 (2012) 073009 [arXiv: 1208.0769] [INSPIRE].

[29] P. Artoisenet, P. de Aquino, F. Maltoni and O. Mattelaer, Unravelling tīh via the Matrix Element Method, Phys. Rev. Lett. 111 (2013) 091802 [arXiv:1304.6414] [INSPIRE].

[30] P. Agrawal, S. Bandyopadhyay and S.P. Das, Dilepton Signatures of the Higgs Boson with Tau-jet Tagging, arXiv:1308.6511 [INSPIRE].

[31] M.R. Buckley, T. Plehn, T. Schell and M. Takeuchi, Buckets of Higgs and Tops, JHEP 02 (2014) 130 [arXiv:1310.6034] [INSPIRE].

[32] M. Farina, C. Grojean, F. Maltoni, E. Salvioni and A. Thamm, Lifting degeneracies in Higgs couplings using single top production in association with a Higgs boson, JHEP 05 (2013) 022 [arXiv:1211.3736] [INSPIRE].

[33] S. Biswas, E. Gabrielli, F. Margaroli and B. Mele, Direct constraints on the top-Higgs coupling from the $8 \mathrm{TeV}$ LHC data, JHEP 07 (2013) 073 [arXiv: 1304.1822] [INSPIRE].

[34] J. Ellis, D.S. Hwang, K. Sakurai and M. Takeuchi, Disentangling Higgs-Top Couplings in Associated Production, JHEP 04 (2014) 004 [arXiv: 1312.5736] [INSPIRE].

[35] C. Englert and E. Re, Bounding the top Yukawa coupling with Higgs-associated single-top production, Phys. Rev. D 89 (2014) 073020 [arXiv: 1402.0445] [INSPIRE].

[36] W.J. Stirling and D.J. Summers, Production of an intermediate mass Higgs boson in association with a single top quark at LHC and SSC, Phys. Lett. B 283 (1992) 411 [INSPIRE].

[37] F. Maltoni, D.L. Rainwater and S. Willenbrock, Measuring the top quark Yukawa coupling at hadron colliders via $t \bar{t} H, H \rightarrow W^{+} W^{-}$, Phys. Rev. D 66 (2002) 034022 [hep-ph/0202205] [INSPIRE]. 
[38] D.E. Morrissey, T. Plehn and T.M.P. Tait, Physics searches at the LHC, Phys. Rept. 515 (2012) 1 [arXiv: 0912.3259] [INSPIRE].

[39] J.R. Ellis, M.K. Gaillard and D.V. Nanopoulos, A Phenomenological Profile of the Higgs Boson, Nucl. Phys. B 106 (1976) 292 [INSPIRE].

[40] M.A. Shifman, A.I. Vainshtein, M.B. Voloshin and V.I. Zakharov, Low-Energy Theorems for Higgs Boson Couplings to Photons, Sov. J. Nucl. Phys. 30 (1979) 711 [inSPIRE].

[41] B.A. Kniehl and M. Spira, Low-energy theorems in Higgs physics, Z. Phys. C 69 (1995) 77 [hep-ph/9505225] [INSPIRE].

[42] T. Plehn, Lectures on LHC Physics, Lect. Notes Phys. 844 (2012) 1 [arXiv:0910.4182] [INSPIRE].

[43] D. Graudenz, M. Spira and P.M. Zerwas, QCD corrections to Higgs boson production at proton proton colliders, Phys. Rev. Lett. 70 (1993) 1372 [INSPIRE].

[44] M. Spira, A. Djouadi, D. Graudenz and P.M. Zerwas, Higgs boson production at the LHC, Nucl. Phys. B 453 (1995) 17 [hep-ph/9504378] [INSPIRE].

[45] M. Krämer, E. Laenen and M. Spira, Soft gluon radiation in Higgs boson production at the LHC, Nucl. Phys. B 511 (1998) 523 [hep-ph/9611272] [INSPIRE].

[46] S. Marzani, R.D. Ball, V. Del Duca, S. Forte and A. Vicini, Higgs production via gluon-gluon fusion with finite top mass beyond next-to-leading order, Nucl. Phys. B 800 (2008) 127 [arXiv:0801.2544] [INSPIRE].

[47] A. Pak, M. Rogal and M. Steinhauser, Finite top quark mass effects in NNLO Higgs boson production at LHC, JHEP 02 (2010) 025 [arXiv:0911.4662] [INSPIRE].

[48] M. Spira, QCD effects in Higgs physics, Fortsch. Phys. 46 (1998) 203 [hep-ph/9705337] [INSPIRE].

[49] U. Baur, T. Plehn and D.L. Rainwater, Measuring the Higgs boson self coupling at the LHC and finite top mass matrix elements, Phys. Rev. Lett. 89 (2002) 151801 [hep-ph/0206024] [INSPIRE].

[50] J. Grigo, J. Hoff, K. Melnikov and M. Steinhauser, On the Higgs boson pair production at the LHC, Nucl. Phys. B $\mathbf{8 7 5}$ (2013) 1 [arXiv:1305.7340] [INSPIRE].

[51] X. Li and M.B. Voloshin, Remarks on double Higgs boson production by gluon fusion at threshold, Phys. Rev. D 89 (2014) 013012 [arXiv:1311.5156] [InSPIRE].

[52] R.K. Ellis, I. Hinchliffe, M. Soldate and J.J. van der Bij, Higgs Decay to $\tau^{+} \tau^{-}$: A Possible Signature of Intermediate Mass Higgs Bosons at the SSC, Nucl. Phys. B 297 (1988) 221 [INSPIRE].

[53] U. Baur and E.W.N. Glover, Higgs Boson Production at Large Transverse Momentum in Hadronic Collisions, Nucl. Phys. B 339 (1990) 38 [InSPIRE].

[54] A. Banfi, A. Martin and V. Sanz, Probing top-partners in Higgs+jets, JHEP 08 (2014) 053 [arXiv: 1308.4771] [INSPIRE].

[55] A. Azatov and A. Paul, Probing Higgs couplings with high $p_{T}$ Higgs production, JHEP 01 (2014) 014 [arXiv: 1309.5273] [INSPIRE].

[56] C. Grojean, E. Salvioni, M. Schlaffer and A. Weiler, Very boosted Higgs in gluon fusion, JHEP 05 (2014) 022 [arXiv:1312.3317] [INSPIRE]. 
[57] E. Bagnaschi, G. Degrassi, P. Slavich and A. Vicini, Higgs production via gluon fusion in the POWHEG approach in the SM and in the MSSM, JHEP 02 (2012) 088 [arXiv:1111.2854] [INSPIRE].

[58] R.V. Harlander, T. Neumann, K.J. Ozeren and M. Wiesemann, Top-mass effects in differential Higgs production through gluon fusion at order $\alpha_{s}^{4}$, JHEP 08 (2012) 139 [arXiv: 1206.0157] [INSPIRE].

[59] R.V. Harlander and T. Neumann, Probing the nature of the Higgs-gluon coupling, Phys. Rev. D 88 (2013) 074015 [arXiv: 1308.2225] [INSPIRE].

[60] C. Englert, M. McCullough and M. Spannowsky, Gluon-initiated associated production boosts Higgs physics, Phys. Rev. D 89 (2014) 013013 [arXiv: 1310.4828] [INSPIRE].

[61] M. Buschmann, C. Englert, D. Goncalves, T. Plehn and M. Spannowsky, Resolving the Higgs-Gluon Coupling with Jets, Phys. Rev. D 90 (2014) 013010 [arXiv:1405.7651] [INSPIRE].

[62] D.S.M. Alves, M.R. Buckley, P.J. Fox, J.D. Lykken and C.-T. Yu, Stops and $\not_{T}$ : The shape of things to come, Phys. Rev. D 87 (2013) 035016 [arXiv:1205.5805] [INSPIRE].

[63] S. Bornhauser, M. Drees, S. Grab and J.S. Kim, Light Stop Searches at the LHC in Events with two b-Jets and Missing Energy, Phys. Rev. D 83 (2011) 035008 [arXiv:1011.5508] [INSPIRE].

[64] N. Desai and B. Mukhopadhyaya, Constraints on supersymmetry with light third family from LHC data, JHEP 05 (2012) 057 [arXiv:1111.2830] [INSPIRE].

[65] Z. Han, A. Katz, D. Krohn and M. Reece, (Light) Stop Signs, JHEP 08 (2012) 083 [arXiv: 1205.5808] [INSPIRE].

[66] G. Bélanger, R.M. Godbole, L. Hartgring and I. Niessen, Top Polarization in Stop Production at the LHC, JHEP 05 (2013) 167 [arXiv: 1212.3526] [INSPIRE].

[67] X.-Q. Li, Z.-G. Si, K. Wang, L. Wang, L. Zhang and G. Zhu, Light Top Squark in Precision Top Quark Sample, Phys. Rev. D 89 (2014) 077703 [arXiv:1311.6874] [INSPIRE].

[68] M. Schlaffer, M. Spannowsky, M. Takeuchi, A. Weiler and C. Wymant, Boosted Higgs Shapes, Eur. Phys. J. C 74 (2014) 3120 [arXiv:1405.4295] [InSPIRE].

[69] N. Kauer and G. Passarino, Inadequacy of zero-width approximation for a light Higgs boson signal, JHEP 08 (2012) 116 [arXiv:1206.4803] [INSPIRE].

[70] F. Caola and K. Melnikov, Constraining the Higgs boson width with ZZ production at the LHC, Phys. Rev. D 88 (2013) 054024 [arXiv:1307.4935] [inSPIRE].

[71] J.M. Campbell, R.K. Ellis and C. Williams, Bounding the Higgs width at the LHC using full analytic results for $g g \rightarrow e^{-} e^{+} \mu^{-} \mu^{+}$, JHEP 04 (2014) 060 [arXiv:1311.3589] [INSPIRE].

[72] J.M. Campbell, R.K. Ellis and C. Williams, Bounding the Higgs width at the LHC: complementary results from $H \rightarrow W W$, Phys. Rev. D 89 (2014) 053011 [arXiv:1312.1628] [INSPIRE].

[73] CMS collaboration, Properties of the Higgs-like boson in the decay $H$ to ZZ to $4 \mathrm{l}$ in $p p$ collisions at $\sqrt{s}=7$ and $8 \mathrm{TeV}$, CMS-PAS-HIG-13-002.

[74] CMS collaboration, Constraints on the Higgs boson width from off-shell production and decay to Z-boson pairs, Phys. Lett. B 736 (2014) 64 [arXiv:1405.3455] [INSPIRE]. 
[75] ATLAS collaboration, Determination of the off-shell Higgs boson signal strength in the high-mass ZZ final state with the ATLAS detector, ATLAS-CONF-2014-042 (2014).

[76] C. Englert and M. Spannowsky, Limitations and Opportunities of Off-Shell Coupling Measurements, Phys. Rev. D 90 (2014) 053003 [arXiv: 1405.0285] [INSPIRE].

[77] J.S. Gainer, J. Lykken, K.T. Matchev, S. Mrenna and M. Park, Beyond Geolocating: Constraining Higher Dimensional Operators in $H \rightarrow 4 \ell$ with Off-Shell Production and More, arXiv: 1403.4951 [INSPIRE].

[78] E.W.N. Glover and J.J. van der Bij, Z boson pair production via gluon fusion, Nucl. Phys. B 321 (1989) 561 [INSPIRE].

[79] A. Azatov, C. Grojean, A. Paul and E. Salvioni, Taming the off-shell Higgs boson, arXiv: 1406.6338 [INSPIRE].

[80] G. Cacciapaglia, A. Deandrea, G. Drieu La Rochelle and J.-B. Flament, Higgs couplings: disentangling New Physics with off-shell measurements, Phys. Rev. Lett. 113 (2014) 201802 [arXiv: 1406.1757] [INSPIRE].

[81] T. Gleisberg et al., Event generation with SHERPA 1.1, JHEP 02 (2009) 007 [arXiv:0811.4622] [INSPIRE].

[82] F. Krauss, R. Kuhn and G. Soff, AMEGIC ++ 1.0: A matrix element generator in $\mathrm{C}++$, JHEP 02 (2002) 044 [hep-ph/0109036] [INSPIRE].

[83] S. Hoeche, F. Krauss, P. Maierhoefer, S. Pozzorini, M. Schonherr and F. Siegert, Next-to-leading order QCD predictions for top-quark pair production with up to two jets merged with a parton shower, arXiv:1402.6293 [INSPIRE].

[84] S. Hoeche, F. Krauss, S. Pozzorini, M. Schoenherr, J.M. Thompson and K.C. Zapp, Triple vector boson production through Higgs-Strahlung with NLO multijet merging, Phys. Rev. D 89 (2014) 093015 [arXiv: 1403.7516] [INSPIRE].

[85] S. Catani, F. Krauss, R. Kuhn and B.R. Webber, QCD matrix elements + parton showers, JHEP 11 (2001) 063 [hep-ph/0109231] [INSPIRE].

[86] S. Hoeche, F. Krauss, S. Schumann and F. Siegert, QCD matrix elements and truncated showers, JHEP 05 (2009) 053 [arXiv: 0903.1219] [INSPIRE].

[87] S. Hoeche, F. Krauss, M. Schonherr and F. Siegert, QCD matrix elements + parton showers: The NLO case, JHEP 04 (2013) 027 [arXiv: 1207.5030] [INSPIRE].

[88] T. Gehrmann, S. Hoche, F. Krauss, M. Schonherr and F. Siegert, NLO QCD matrix elements + parton showers in $e^{+} e^{-} \rightarrow$ hadrons, JHEP 01 (2013) 144 [arXiv:1207.5031] [INSPIRE].

[89] S. Hoeche, F. Krauss and M. Schonherr, Uncertainties in MEPS@NLO calculations of $h+$ jets, Phys. Rev. D 90 (2014) 014012 [arXiv:1401.7971] [INSPIRE].

[90] F. Cascioli, P. Maierhofer and S. Pozzorini, Scattering Amplitudes with Open Loops, Phys. Rev. Lett. 108 (2012) 111601 [arXiv:1111.5206] [INSPIRE].

[91] A. Denner, S. Dittmaier and L. Hofer, COLLIER - A fortran-library for one-loop integrals, PoS (LL2014) 071 [arXiv: 1407.0087] [INSPIRE].

[92] A. Denner and S. Dittmaier, Scalar one-loop 4-point integrals, Nucl. Phys. B 844 (2011) 199 [arXiv: 1005.2076] [INSPIRE].

[93] A. Denner and S. Dittmaier, Reduction of one loop tensor five point integrals, Nucl. Phys. B 658 (2003) 175 [hep-ph/0212259] [INSPIRE]. 
[94] J. Alwall, Q. Li and F. Maltoni, Matched predictions for Higgs production via heavy-quark loops in the SM and beyond, Phys. Rev. D 85 (2012) 014031 [arXiv:1110.1728] [InSPIRE].

[95] R. Frederix et al., Higgs pair production at the LHC with NLO and parton-shower effects, Phys. Lett. B 732 (2014) 142 [arXiv:1401.7340] [InSPIRE].

[96] F. Maltoni, E. Vryonidou and M. Zaro, Top-quark mass effects in double and triple Higgs production in gluon-gluon fusion at NLO, JHEP 11 (2014) 079 [arXiv:1408.6542] [INSPIRE].

[97] S. Frixione and B.R. Webber, Matching NLO QCD computations and parton shower simulations, JHEP 06 (2002) 029 [hep-ph/0204244] [INSPIRE].

[98] S. Hoeche, F. Krauss, M. Schonherr and F. Siegert, A critical appraisal of $N L O+P S$ matching methods, JHEP 09 (2012) 049 [arXiv:1111.1220] [INSPIRE].

[99] S. Hoeche, F. Krauss, M. Schonherr and F. Siegert, $W+n$-jet predictions at the Large Hadron Collider at next-to-leading order matched with a parton shower, Phys. Rev. Lett. 110 (2013) 052001 [arXiv: 1201.5882] [INSPIRE].

[100] T. Sjöstrand, S. Mrenna and P.Z. Skands, PYTHIA 6.4 Physics and Manual, JHEP 05 (2006) 026 [hep-ph/0603175] [INSPIRE].

[101] J. Alwall, M. Herquet, F. Maltoni, O. Mattelaer and T. Stelzer, MadGraph 5: Going Beyond, JHEP 06 (2011) 128 [arXiv:1106.0522] [INSPIRE].

[102] J.M. Campbell, R.K. Ellis, R. Frederix, P. Nason, C. Oleari and C. Williams, NLO Higgs Boson Production Plus One and Two Jets Using the POWHEG BOX, MadGraph4 and MCFM, JHEP 07 (2012) 092 [arXiv:1202.5475] [INSPIRE].

[103] J.M. Campbell, R.K. Ellis and C. Williams, MCFM - Monte Carlo for FeMtobarn processes, http://mcfm.fnal.gov.

[104] F. Campanario, M. Kubocz and D. Zeppenfeld, Gluon-fusion contributions to $\Phi+2$ Jet production, Phys. Rev. D 84 (2011) 095025 [arXiv: 1011.3819] [InSPIRE].

[105] M. Cacciari, G.P. Salam and G. Soyez, The Anti-kt jet clustering algorithm, JHEP 04 (2008) 063 [arXiv: 0802.1189] [INSPIRE].

[106] M. Cacciari, G.P. Salam and G. Soyez, FastJet User Manual, Eur. Phys. J. C 72 (2012) 1896 [arXiv:1111.6097] [INSPIRE].

[107] M. Grazzini and H. Sargsyan, Heavy-quark mass effects in Higgs boson production at the LHC, JHEP 09 (2013) 129 [arXiv: 1306.4581] [inSPIRE].

[108] M. Wiesemann, R. Frederix, S. Frixione, V. Hirschi, F. Maltoni and P. Torrielli, Higgs production in association with bottom quarks, arXiv:1409.5301 [INSPIRE].

[109] R.V. Harlander, H. Mantler and M. Wiesemann, Transverse momentum resummation for Higgs production via gluon fusion in the MSSM, JHEP 11 (2014) 116 [arXiv:1409.0531] [INSPIRE].

[110] S. Catani and M.H. Seymour, A general algorithm for calculating jet cross-sections in NLO QCD, Nucl. Phys. B 485 (1997) 291 [Erratum ibid. B 510 (1998) 503] [hep-ph/9605323] [INSPIRE].

[111] S. Schumann and F. Krauss, A parton shower algorithm based on Catani-Seymour dipole factorisation, JHEP 03 (2008) 038 [arXiv:0709.1027] [INSPIRE].

[112] T. Plehn, D.L. Rainwater and D. Zeppenfeld, Determining the structure of Higgs couplings at the LHC, Phys. Rev. Lett. 88 (2002) 051801 [hep-ph/0105325] [INSPIRE]. 
[113] C. Ruwiedel, N. Wermes and M. Schumacher, Prospects for the measurement of the structure of the coupling of a Higgs boson to weak gauge bosons in weak boson fusion with the ATLAS detector, Eur. Phys. J. C 51 (2007) 385 [InSPIRE].

[114] G. Klamke and D. Zeppenfeld, Higgs plus two jet production via gluon fusion as a signal at the CERN LHC, JHEP 04 (2007) 052 [hep-ph/0703202] [INSPIRE].

[115] K. Hagiwara, Q. Li and K. Mawatari, Jet angular correlation in vector-boson fusion processes at hadron colliders, JHEP 07 (2009) 101 [arXiv: 0905.4314] [INSPIRE].

[116] C. Englert, D. Goncalves-Netto, K. Mawatari and T. Plehn, Higgs Quantum Numbers in Weak Boson Fusion, JHEP 01 (2013) 148 [arXiv: 1212.0843] [INSPIRE].

[117] C. Englert, D. Goncalves, G. Nail and M. Spannowsky, The shape of spins, Phys. Rev. D 88 (2013) 013016 [arXiv: 1304.0033] [INSPIRE].

[118] K. Hagiwara and S. Mukhopadhyay, Azimuthal correlation among jets produced in association with a bottom or top quark pair at the LHC, JHEP 05 (2013) 019 [arXiv:1302.0960] [INSPIRE].

[119] M.R. Buckley, T. Plehn and M.J. Ramsey-Musolf, Top squark with mass close to the top quark, Phys. Rev. D 90 (2014) 014046 [arXiv: 1403.2726] [inSPIRE].

[120] J. Pumplin, D.R. Stump, J. Huston, H.L. Lai, P.M. Nadolsky and W.K. Tung, New generation of parton distributions with uncertainties from global QCD analysis, JHEP 07 (2002) 012 [hep-ph/0201195] [INSPIRE].

[121] G. Passarino, Higgs CAT, Eur. Phys. J. C 74 (2014) 2866 [arXiv:1312.2397] [InSPIRE].

[122] M. Bonvini, F. Caola, S. Forte, K. Melnikov and G. Ridolfi, Signal-background interference effects for $g g \rightarrow H \rightarrow W^{+} W^{-}$beyond leading order, Phys. Rev. D 88 (2013) 034032 [arXiv: 1304.3053] [INSPIRE].

[123] N. Cabibbo and A. Maksymowicz, Angular Correlations in $\mathrm{Ke}_{4}$ Decays and Determination of Low-Energy $\pi-\pi$ Phase Shifts, Phys. Rev. 137 (1965) B438 [Erratum ibid. 168 (1968) 1926] [INSPIRE].

[124] J.R. Dell'Aquila and C.A. Nelson, $P$ or $C P$ Determination by Sequential Decays: $V_{1} V_{2}$ Modes With Decays Into $\bar{\ell}_{A} \ell_{B}$ And/or $\bar{q}_{A} q_{B}$, Phys. Rev. D 33 (1986) 80 [InSPIRE].

[125] J.R. Dell'Aquila and C.A. Nelson, Distinguishing a Spin 0 Technipion and an Elementary Higgs Boson: $V_{1} V_{2}$ Modes With Decays Into $\bar{\ell}_{A} \ell_{B}$ And/or $\bar{q}_{A} q_{B}$, Phys. Rev. D 33 (1986) 93 [INSPIRE].

[126] C.A. Nelson, Correlation Between Decay Planes in Higgs Boson Decays Into W Pair (Into Z Pair), Phys. Rev. D 37 (1988) 1220 [inSPIRE].

[127] M. Klute, R. Lafaye, T. Plehn, M. Rauch and D. Zerwas, Measuring Higgs Couplings at a Linear Collider, Europhys. Lett. 101 (2013) 51001 [arXiv:1301.1322] [INSPIRE]. 